\title{
Antimicrobial activities of the extracts from bark of ironwood (Mesua ferrea) and their role in production of traditional sugar palm wine
}

\author{
Nguyen Thi Dong Phuong, Dang Minh Nhat \\ The University of Da Nang - University of Science And Technology, 54 Nguyen Luong Bang street, Da Nang, Viet Nam
}

Email address:

nhatdangminh@gmail.com (D. M. Nhat)

To cite this article:

Nguyen Thi Dong Phuong, Dang Minh Nhat. Antimicrobial Activities of the Extracts from Bark of Ironwood (Mesua ferrea) and their Role in Production of Traditional Sugar Palm Wine. Journal of Food and Nutrition Sciences. Special Issue: Food Processing and Food Quality. Vol. 3, No. 1-2, 2015, pp. 65-69. doi: 10.11648/j.jfns.s.2015030102.22

\begin{abstract}
Bark from ironwood (chuồn in Vietnamese) is used in the production of traditional sugar palm wine of ethnic minorities in Central Vietnam. The objectives of this study were to investigate the antimicrobial properties of water and alcohol extracts of this bark and to determine the major active chemical components in these extracts. The agar well diffusion test was used to evaluate the antimicrobial activities of water and ethanol $(10 \% \mathrm{v} / \mathrm{v}$ and $30 \% \mathrm{v} / \mathrm{v})$ extracts of ironwood bark against Escherichia coli, Staphylococcus aureus, Acetobacter xylinum and Saccharomyces cerevisiae. All the extracts were found to possess significant inhibitory property against the tested microorganisms except $S$. cerevisae. The higher antimicrobial properties were noticed in the ethanol extracts compared to that of the water extract. Such antimicrobial property of the bark could explain its important role in supporting spontaneous alcohol fermentation of sugar palm sap in traditional wine making by ethnic minorities in Central Vietnam. The study also revealed the presence of saponins, tannins, phenols, terpenoids and flavonoids in the bark extracts. The analysis of flavonoid content in the ironwood bark gave the result of $15.57 \%$ (on dry basis), these flavonoids were also found to possess stronger antimicrobial activities than those of the ethanol extracts.
\end{abstract}

Keywords: Ironwood Bark Extracts, Antimicrobial Activities, Escherichia Coli, Staphylococcus Aureus, Acetobacter Xylinum, Saccharomyces cerevisiae, Traditional Sugar Palm Wine

\section{Introduction}

Finding new bioactive compounds from natural sources for preparation of food additives, functional foods or for medicinal purposes has been the target of many studies, attracting great attention from scientists as well as the public media worldwide recently $[1,2]$. According to the WHO report, medicinal plants are the best sources in pharmaceutical production. About $80 \%$ of the population in developing countries has been using medicine of plant origin. Hence this raises the need of extensive studies on the chemical composition, functional and safety properties of these medicinal plants [3].

Various phytochemicals have an ability to protect plants from pathogens and they also could be exploited for human uses $[2,4]$. Tannins, which are compounds associated with protein synthesis, have been shown to possess antimicrobial activities [1, 5, 8]. The effect of flavonoids, a plant polyphenolic hydroxylate, against pathogenic microorganisms in vivo and in vitro has been well documented. Terpenoids could also have good antimicrobial capability beside their main role in aroma formation. Saponins, which are glycosides, have been shown to have ability to inhibit the Gram positive bacteria such as Streptococcus aureus [2]. All these compounds are from natural origins and therefore they have much more advantages over synthetic antimicrobial agents in terms of consumer safety.

Tropical jungles in Vietnam has diversifying flora, including those plants which have potential for retrieving bioactive compounds for human uses but are not very well studied. Among them there is ironwood (Mesua ferrea) (named "chuồn" in Vietnamese), which is found abundant in the mountainous area of Central Vietnam. The bark from the tree has been used traditionally by the ethnic minorities in making sugar palm wine. Accordingly, the dry bark is added to the sap from sugar palm to prevent the spoilage during spontaneous fermentation and to give the characteristic taste for fermented product. 
We believe the bark of ironwood has the special ability to inhibit the growth of undesirable microorganisms, but support the alcohol fermentation of the sugar palm sap. Therefore, in this study we investigated the inhibitory effect of water and ethanol extract of the ironwood bark against Escherichia coli, Staphylococcus aureus, Acetobacter xylinum and Saccharomyces cerevisiae which are representatives for Gram positive and Gram negative bacteria, acetic acid bacteria, ethanol forming yeasts. Another objective of the study was also to determine the presence of some bioactive compounds in the extracts tested.

\section{Materials and Methods}

\subsection{Preparation of Water and Ethanol Extracts from Ironwood Bark}

The bark of ironwood was cut from the middle trunk of the trees grown in the jungle of Quảng Nam province, Vietnam. It was then dried and ground into powder. Each $10 \mathrm{~g}$ of the bark powder was suspended in $100 \mathrm{ml}$ solvent contained in closed flask and let extracted 3 days in shaking device at 70 $\mathrm{rpm}$. After that, the extract was filtered and evaporated to the final volume of $30 \mathrm{ml}$. Solvents used for extraction were distilled water, ethanol 10\% (v/v) and 30\% (v/v).

\subsection{Preparation of Microorganism Cultures}

Tested microbial species including Escherichia coli ATCC 25922, Staphylococcus aureus ATCC 25923, Saccharomyces cerevisiae were donated by National Agro-Forestry-Fisheries Quality Assurance Department Zone 2 in Da Nang, Vietnam. Acetobacter xylinum (acetic acid bacterium) was supplied by Biotechnological Department of Da Nang University of Technology.

E.coli and S.aureus were grown on Brain Heart Infusion (BHI) agar for $24 \mathrm{~h}$ at $37^{\circ} \mathrm{C}$, A. xylinum at $30^{\circ} \mathrm{C}$ for $36 \mathrm{~h}$. Hansen agar was used for growing $S$. cerevisiae at $30^{\circ} \mathrm{C}$ for 36h. These species were then cultured in respective liquid media for next experiments.

\subsection{Agar Well Diffusion Assay}

Antimicrobial activity was determined by using the agar well diffusion method. Prior to streaking $100 \mu \mathrm{l}$ microbial suspension on the Mueller Hinton plates, 2 wells of $8 \mathrm{~mm}$ in diameter were punched by means of a sterile borer. In each plate, $100 \mu \mathrm{l}$ of the test extract was introduced into one well, $100 \mu \mathrm{l}$ distilled water was introduced into the other as a control. The plates were incubated overnight at $37^{\circ} \mathrm{C}$. The antimicrobial effect was evaluated by measuring the diameter of inhibition zone. The width of inhibition zone was calculated as $(D-d)$, where $D$ was the zone diameter $(\mathrm{mm})$ and $d$ was the well diameter (mm).

\subsection{Qualitative Analysis of Some Active Compounds}

- Determination of the presence of saponin (Frothing Test) [6]: by vigorously shaking $3 \mathrm{ml}$ extracts in test tube.
Stable foams likely indicate the presence of saponin.

- Determination of the presence of phenols and tannins (Ferric Chloride Test) [6]: by pipetting $1 \mathrm{ml}$ of the bark extracts and $2 \mathrm{ml}$ of solution $\mathrm{FeCl}_{3} 2 \%$. The appearance of dark green color is typical for the possible presence of phenols or tannins.

- Determination of the presence of terpenoids (Salkowski's Test) [6]: The content of $1 \mathrm{ml}$ the extracts, $2 \mathrm{ml}$ conc. $\mathrm{H}_{2} \mathrm{SO}_{4}$ in test tube was gently shaken. The change of color to brown red was indicative for the presence of terpenoids.

- Determination of the presence of flavonoids (Shinoda Test) [9]: One ml of the extract, magnesium powder and a few drops of conc. $\mathrm{HCl}$ were poured into a test tube. The presence of flavonoids was likely to be confirmed by the appearance of orange, red, pink or purple color.

- Determination of the presence of glycosides (Keller Killiani Test) [2]: One $\mathrm{ml}$ of the extract, $2 \mathrm{ml}$ of dilute acetic acid, 2 drops $\mathrm{FeCl}_{3} 2 \%$ and $2 \mathrm{ml}$ of the conc. $\mathrm{H}_{2} \mathrm{SO}_{4}$ were taken into a test tube. The formation of brown color was indicative for the presence of glycosides

\subsection{Extraction and Quantitative Determination of Flavonoids (Talli Procedure) [9]}

Exactly $5 \mathrm{~g}$ of ground bark was subjected to Soxhlet extraction firstly with petroleum ether to eliminate lipids, colorants, followed by drying to remove the solvent out of the bark sample and then the bark was extracted again with ethanol $96 \%(\mathrm{v} / \mathrm{v})$. The extract was dried in a rotary evaporator and the residual fraction was dissolved in $100 \mathrm{ml}$ distilled water. The solution was further extracted with ethyl acetate for several times until the negative result for the presence of flavonoids evolved. Ethyl acetate was then dried off to obtain the residue at $80^{\circ} \mathrm{C}$ to constant weight $\mathrm{m}(\mathrm{g})$. The content of crude flavonoids was calculated as follows:

$$
\% \text { flavonoids }=\frac{\mathrm{m}}{\mathrm{a} \times(100-\mathrm{X})} \times 100
$$

$\mathrm{m}$ : Weight of obtained crude flavonoids $(\mathrm{g})$

a: Weight of ground bark, $5 \mathrm{~g}$

$\mathrm{X}$ : Moisture content of the bark (\%).

\subsection{Data Analysis}

Means and standard deviations were calculated from the results of 3 replicates. Least Significant Difference (LSD) Test and unpaired t-test (at $\alpha=5 \%$ ) were used for data analysis.

\section{Results and Discussions}

\subsection{Antimicrobial Activities of the Water and Ethanol Extracts from Ironwood Bark}

The water and ethanol $(10 \% \mathrm{v} / \mathrm{v}$ and $30 \% \mathrm{v} / \mathrm{v})$ extracts were prepared according to the method described in 2.1 . 
Antimicrobial effect of these extracts was determined using agar well diffusion assay as mentioned above. The results are presented in Table 1 as mean \pm standard deviation of triplicates. Figure 1 illustrates the inhibition zones formed by the effect of the ethanol extracts in agar dishes.
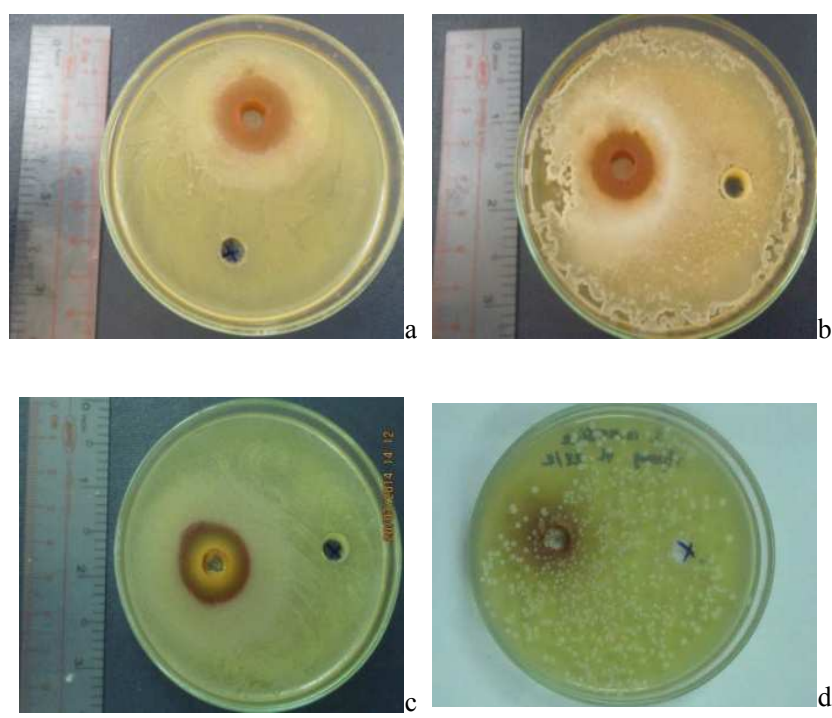

Figure 1. Inhibition zones influenced by ethanol 30\% (v/v) extracts against (a) E. coli, (b) S. aureus, (c) A.xylinum and (d) S.cerevisiae

Table 1. Antimicrobial activities of ironwood bark extracts

\begin{tabular}{lcccc}
\hline \multirow{2}{*}{ Extract } & \multicolumn{3}{c}{ (D-d) $(\mathbf{m m})$} \\
\cline { 2 - 5 } & E. coli & S. aureus & A. xylinum & S. cerevisiae \\
\hline EtOH $30 \%(\mathrm{v} / \mathrm{v})$ & $11,67^{\mathrm{b}} \pm 0,50$ & $11,00^{\mathrm{c}} \pm 0,24$ & $12,50^{\mathrm{b}} \pm 0,44$ & 0 \\
EtOH $10 \%(\mathrm{v} / \mathrm{v})$ & $11,50^{\mathrm{b}} \pm 0,50$ & $9,33^{\mathrm{b}} \pm 0,85$ & $12,33^{\mathrm{b}} \pm 0,75$ & 0 \\
Water & $9,33^{\mathrm{a}} \pm 0,48$ & $4,33^{\mathrm{a}} \pm 0,50$ & $5,33^{\mathrm{a}} \pm 0,48$ & 0 \\
Control & 0 & 0 & 0 & 0 \\
\hline
\end{tabular}

Note:- D, d: diameter of the inhibition zone and the well, respectively;

- Different letters associated with the values in each column indicate significant difference at $\alpha=5 \%$.

The results showed that all the extracts exhibited strong antimicrobial effect against $E$. coli, $S$. aureus and A. xylinum, since clear inhibition zones around those agar wells were noticed with the addition of the extracts in contrast with the absence of clear zone around the well with control samples (water). However, none of the extracts was found to inhibit the growth of $S$. cerevisiae. This effect depended on the various types of extracts and microbial species. In general, the water extracts showed weaker antimicrobial activities than the ethanol extracts. LSD Test showed significant differences $(\alpha=5 \%)$ between samples with water extracts and with ethanol extracts. This suggested that ethanol 10\% (v/v) and 30\% (v/v) were better solvents for extraction active compounds from the ironwood bark powder. The ethanol $10 \%(\mathrm{v} / \mathrm{v})$ and ethanol $30 \%(\mathrm{v} / \mathrm{v})$ extracts made no difference in diameter of the inhibition zones except the case of $S$. aureus whereas the ethanol $30 \%$ showed a greater effect. Water extracts of ironwood bark inhibited $E$. coli rather well, but the effect was weaker against $S$. aureus and A. xylinum. In all cases, $S$. cerevisiae could grow well in the media added with the ironwood bark extracts.

The better ability of aqueous ethanol to extract antimicrobial components from plant sources was also reported in many studies. Shinkafi et al. [7] found out that the ethanol extracts of onion exhibited high activity against pathogenic microorganisms, whereas the water extracts didn't have such ability. In another study, Bipul Biswas et al. [2] confirmed the antimicrobial effect of the ethanol extract of guava leaves, but water could not extract antimicrobial compounds from guava leaves. This result suggests that aqueous ethanol could be a good solvent for extracting antimicrobial components from plants.

The extracts of ironwood bark were effective against wide spectrum of bacteria, either Gram positive or Gram negative bacteria. We deduced that there could be various antimicrobial compounds in the extracts, acting against different bacterial species. Strong inhibition effect of the ironwood bark extracts against Acetobacter xylinum, which was demonstrated by the large inhibition zones in the agar dishes, is very meaningful in the traditional method of making palm wine used by ethnic minorities in Central Vietnam. During the spontaneous fermentation of the palm sap, low content of alcohol formed in the sap should be ideal for the growth of acetic acid bacteria. These bacteria could lower the alcohol content of the product as well as raise the acetic acid level which makes the taste of the product unacceptable. Therefore, adding the bark of ironwood into the sap of sugar palm, the local people have allowed the 
active antimicrobial components to extract into water and later in the alcohol media of the sap and therefore protect the product from undesirable microorganisms, while allows alcohol forming yeast such as $S$. cerevisiae to ferment at their full capacity. This theory suits the experiences of the local people. According to them, without addition of the bark, the product is spoilt with unacceptable taste, while the presence of the bark could extend the shelf life of the product for couple of days.

\subsection{Qualitative Analysis of Active Compounds in the Extracts of Ironwood Bark}

In this study, we aimed to find out the presence of some active compounds in the water and ethanol $(10 \% \mathrm{v} / \mathrm{v})$ extracts using characteristic reactions with the reagents mentioned in section 2.4. Saponin, terpenoids, flavonoids, glycosides, phenols and tannins were our targets, because they have been believed to have antimicrobial activities. The results were presented in Table 2.

Table 2. The presence of some active compounds in water and ethanol extracts

\begin{tabular}{lccccc}
\hline Solvent & Saponins & Phenols and tannins & Terpenoids & Flavonoids & Glycosides \\
\hline Ethanol & - & + & + & + & + \\
Water & + & + & + & + & + \\
\hline
\end{tabular}

Note: $(+)$ presence; (-) absence

From this table, we can see just one difference in composition of two kinds of extracts - the absence of saponins in water extract of the bark, whereas other active components were present in both water and ethanol extracts. Saponins are surface active compounds, which reduce surface tension of solutions and hence facilitate foam formation. Saponins were also reported to possess the inhibition potential against Gram positive bacteria. Their absence in water extract may explain for the lower antimicrobial action against $S$. aureus, what was found in the agar well diffusion assays mentioned above. Phenols, tannins, terpenoids, flavonoids and glycosides are supposed to have potential of antimicrobial effect, their presence in both ethanol and water extracts could be an explanation for the results given in section 3.1 .

\subsection{Quantitative Analysis of Flavonoids in Ironwood Bark and Their Antimicrobial Effect}

Flavonoids were found present in the ironwood bark extracts. These compounds could have various biofunctional properties [10]. In this study, we were interested in their content in the bark of ironwood and their antimicrobial function.

Flavonoids were extracted and determined according to Talli procedure described in 2.5. The results of moisture and flavonoids analysis were shown in Table 3:

Table 3. Analysis of moisture and flavonoids of ironwood bark

\begin{tabular}{ll}
\hline Content & \% \\
\hline Moisture & 6.26 \\
Flavonoids & 15.57 \\
\hline
\end{tabular}

$1 \mathrm{~g}$ of the extracted flavonoids was dissolved in $10 \mathrm{ml}$ of distilled water to make a solution of $10 \%(\mathrm{w} / \mathrm{v})$. Using agar well diffusion assay, the antimicrobial activity of the flavonoid solution against $E$. coli and $S$. aureus was evaluated. Water was used instead of flavonoids in control samples. The results of inhibition zone measurements were presented in Table 4. Figure 2 illustrates inhibition zones formed by flavonoids in agar dishes.
Table 4. Antimicrobial activity of ironwood flavonoids

\begin{tabular}{cccc}
\hline Sample & E. coli & S. aureus & Control \\
\hline$(\mathrm{D}-\mathrm{d})(\mathrm{mm})$ & $17.25 \pm 0.85$ & $16.63 \pm 0.75$ & 0 \\
\hline
\end{tabular}
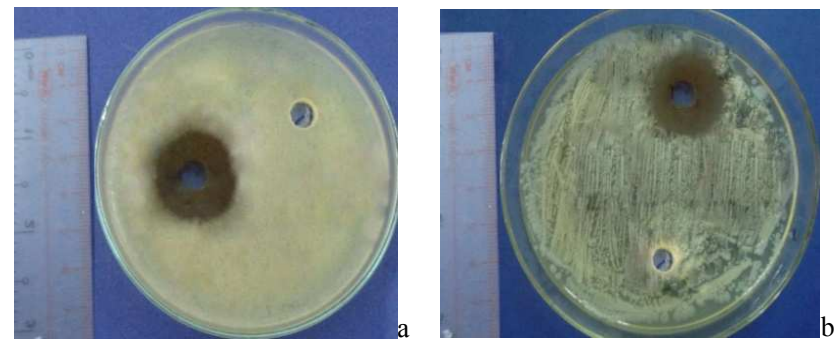

Figure 2. Inhibition zones formed by flavonoids against (a) E. coli; (b) S. aureus.

The results from Table 4 show strong inhibition effect of flavonoids from the ironwood bark against the tested Gram positive and Gram negative bacteria. The differences (D-d) in this case were even better than in the case of using the ethanol extracts mentioned in 3.1. This suggests that the extracted flavonoids at $10 \%(\mathrm{w} / \mathrm{v})$ possessed stronger antimicrobial property against $E$. coli and $S$. aureus than the ethanol extracts of ironwood bark. The result of t-test showed no significant difference in the effect of samples with $E$. coli and $S$. aureus $(p=0.413)$.

Our results could reveal close link between antibacterial of the water and ethanol extracts of ironwood bark with the flavonoid content. The ironwood bark with high content of flavonoids apparently has high capacity to inhibit growth of either $\mathrm{G}+$ or G- bacteria.

\section{Conclusions}

Natural active compounds can play an important role in modern production of food preservatives, functional foods or other human applications. The results of our study showed that the bark of ironwood could be a potential source for extracting antimicrobial components. The bark possibly contains various 
groups of active compounds such as flavonoid, phenols, tannins, saponins, glycosides, terpenoids which generally have antimicrobial effect. The content of flavonoids in the bark $(15.57 \%$ d.b.) is high and these flavonoids have been shown to have excellent antimicrobial properties against both Gram positive and Gram negative bacteria.

The water and ethanol extracts of ironwood bark have been proven to have a unique antimicrobial properties, which would play important role in production of sugar palm wine in Central Vietnam. These extracts exhibited positive antimicrobial effects against Gram positive and Gram negative bacteria, acetic acid bacteria, but did not inhibit the growth of $S$. cerevisiae. The presence of ironwood bark, sugar palm sap could spontaneously be fermented in favor of forming ethanol by S.cerevisiae.

In order to completely understand antimicrobial activities of ironwood bark and possible applications in practice, further work need to be done. These include investigating the effect on wider spectrum of microbial species, identifying main active compounds, and finally evaluating toxicity of the bark or their extracts.

\section{References}

[1] Akiyama H., Fujii K., Yamasaki O., Oono T., and Iwatsuki K. (2001). Antibacterial action of several tannins against Staphylococcus aureus. Journal of Antimicrobial Chemotherapy, 48 (4), 487-491.

[2] Bipul Biswas, Kimberly Rogers, Fredrick McLaughlin, Dwayne Daniels, and Anand Yadav (2013). Antimicrobial activities of leaf extracts of guava (Psidium guajava l.) on two gram-negative and gram-positive bacteria. International Journal of Microbiology, 2013, 1-7.

[3] Gislene G. F. Nasciment; Juliana Locatelli; Paulo C. Freitas; Giuliana L. Silva (2000). Antibacterial activity of plant extracts and phytochemicals on antibiotic-resistant bacteria. Brazilian Journal of Microbiology, 31 (4), 247-256.

[4] Jigna Parekh, Sumitra V. Chanda (2007). In vitro antimicrobial activity and phytochemical analysis of some Indian medicinal plants. Turk Journal of Biology, 31, 53-58.

[5] Min, B. R., Pinchak, W. E., Merkel, R., Walker, S., Tomita, and Anderson, R. C. (2008). Comparative antimicrobial activity of tannin extracts from perennial plants on mastitis pathogens. Scientific Research and Essay 3 (2), 066-073.

[6] Saxena Mamta, Saxena Jyoti (2012). Phytochemical screening of Acorus calamus and Lantana camara. International Research Journal of Pharmacy, 3 (5), 324-326.

[7] Shinkafi, S. A and Dauda, H (2013). Antibacterial activity of Allium cepa (onion) on some pathogenic bacteria associated with ocular infections. Scholars Journal of Applied Medical Sciences, 1(3), 147-151.

[8] Tanaka T., Ishida N., Ishimatsu M., Nonaka G., and Nishioka I. (1992). Tannins and related compounds. Six new complex tannins, guajavins, psidinins and psiguavin from the bark of Psidium guajava L.. Chemical and Pharmaceutical Bulletin, 40(8), 2092-2098.

[9] Vo Thi Mai Huong, Tran Thanh Phong (2013). Some biochemical composition and the antibacterial activity of Morinda citrifolia $L$. extract. In proceeding "The 5-th national conference on ecology and biological resources", pp 10731078.

[10] http://en.wikipedia.org/wiki/Flavonoid. Date 01/06/2014. 